\title{
Aspectos fenomenológicos y éticos del envejecimiento y la demencia
}

\author{
Otto Dörr Zegers* \\ Phenomenological and ethical \\ aspects of aging and dementia
}

Aging is a difficult stage in human life, due to the limitations it imposes and the nearness to death. Modern society tends to be prejudiced against oldness. When dementia is also present, prejudices can be transformed into rejection. The aim of this author tries to devise a phenomenological description of oldness and points out its potentially positive aspects. Following Scheller's classification of dementias (1965), this author tries to determine the common elements of their different forms, using the phenomenological method, which can be applied either to one or to many cases of aging or dementia, not being the number of observations the fundament of the study, as in the case of empirical sciences. Three essential features characterize the spatiality of aging: distance, reduction of mobility and coexistence of the essential and the tangential; temporality is characterized by slowing, coexistence of essential and trivial elements and predominance of present over future and past. In the case of dementias, all characteristics attributed to senescence are exaggerated and/or deformed, for example, «distance» is transformed into indifference or «lowing» becomes a paralysis of temporal development. Now, regarding the main forms of dementia, this author sees their common feature in the loss of the Ego's ability to rise above the immediate situation, to be able to reflect on the environment and on him or herself. This wide vision both of aging and of the dementia process enables the author to identify the prejudices weighing over old age that must be overcome, as well as the reasons to respect and take care of the elderly when they are caught in a process of deterioration (Rev Méd Chile 2005; 133: 113-20).

(Key-w ords: Aging; Dementia; Ethics, medical)

Recibido el 31 de agosto, 2004.

*Profesor Titular de Psiquiatría de la Universidad de Chile, Jefe de Servicio del Hospital Psiquiátrico de Santiago.

A ntes de abordar el tema de las implicaciones éticas del envejecimiento y la demencia es necesario reflexionar sobre lo que estos estados o procesos son en su esencia. Nuestra aproximación será fenomenológica; intentaremos definir estos procesos desde sí mismos y no desde sus causalidades biológicas, como podría ser la esclerosis de los vasos cerebrales y los consiguientes infartos, en la demencia vascular, o la acumulación de amiloide en la enfermedad de Alzheimer.

Correspondencia a: Otto Dörr Zegers. Depto. Psiquiatría de la Universidad de Chile. Facultad de Medicina, Universidad Diego Portales. Fax: 4793578, E-mail: fdeor@@entelchile.net

\section{FenOMENOLOGÍA DEL ENVEJECIMIENTO}

Tanto en castellano como en francés existen dos expresiones para referirse a la también llamada «ercera edad» senescencia y senilidad. La primera apunta al proceso de envejecimiento normal y la segunda, a sus formas patológicas o procesos demenciales. Esta distinción es importante, por cuanto la senescencia encierra posibilidades de compensación, mientras que en la senilidad, como afirma Yves Pelicier ${ }^{1}$, «el organismo se abandona al proceso deficitario» Las posibilidades de compensación que tiene la senescencia, hacen 
imperativo eliminar los prejuicios con que nuestra sociedad occidental sobrecarga todavía a los ancianos. Una forma de acercarnos al mundo del anciano, sería determinar las características de su espacialidad y de su temporalidad -radicales antropológicos que muestran rasgos diferentes en las distintas etapas de la vida-, que ya iniciamos en un trabajo anterior ${ }^{2}$.

\section{a) La espacialidad de la senescencia}

El espacio humano poco o nada tiene que ver con el espacio de las dimensiones, ese que mide en centímetros, metros o kilómetros. Mucho más cerca se encuentra ese amigo que vive en París, a miles de kilómetros, según el espacio geográfico, que mi vecino, de quien me separan, según ese mismo espacio, sólo algunos centímetros de pared. Ahora bien, el existente humano es espacial desde su estructura misma como ser-en-el-mun$\mathrm{do}^{3}$, pero al mismo tiempo está permanentemente regalando espacio»(ein-räumen), dejando a las cosas ser en su espacialidad. Sólo así es posible el conocimiento de las cosas y en el caso de los otros, es ese otorgar espacio la base del respeto, de la amistad y del amor. El espacio de la violencia es lo contrario del espacio amoroso, porque en él la lucha por el espacio vital (en la cual se enmarca también el mundo de la competencia profesional y comercial) se lleva al extremo del desplazamiento y la destrucción. Pero, así como hay un espacio propio del trabajo, otro del amor y otro de la violencia, existen muchos otros espacios humanos, como el religioso, el artístico o el matemático y, según las etapas de la vida, podemos distinguir al menos un espacio infantil, uno adulto y otro propio de la vejez. ¿Cuáles serían las características de este último?

1으 Elejamiento: a diferencia del niño, que vive pegado a lo inmediato y del adulto, que vive lanzado en determinados proyectos de corto y mediano plazo, el anciano vive en un mundo de recogimiento o «oma de distancia». Esto no se refiere sólo al hecho de su abandono del trabajo, sino también a un alejarse de toda participación activa en la vida. La ambición, el afán de poder y el deseo, están en mayor o menor medida apagados. Ahora bien, esto no significa necesariamente pasividad o pereza, sino más bien un nuevo modo de estar en el mundo, un esperar tranquilo lo que la vida pueda regalar, sin tener que conseguirlo sobre la base del trabajo y del esfuerzo. La descripción de la conducta de los ancianos sentados en los parques es muy ilustrativa de este distinto modo de estar en el mundo. A diferencia de los niños, con su permanente inquietud y de los jóvenes que pronto se cansan de la inactividad, los ancianos pueden permanecer largo tiempo sentados en una actitud de contemplación tranquila de lo que ocurre alrededor. Ellos siguen sólo con leves cambios de posición de la cabeza el movimiento de la vida a su alrededor (el juego de los niños, el pasar de los caminantes, los ruidos y las conversaciones), mientras el tronco permanece en una suerte de inmovilidad. Los acontecimientos a su alrededor, son vividos por el anciano como si ocurrieran en un escenario y de ahí esa aparente contradicción entre el gran interés que les despierta cualquier cosa, por banal que ella sea y su casi inmovilidad. Esta actitud de alejamiento de todo se va acentuando en la vejez, hasta llegar, en los confines de la muerte, a una suerte de transfiguración del cuerpo» ¡Cuántas veces hemos presentido la muerte de algún ser querido o conocido, de edad avanzada, sólo porque ese modo de estar alejado se había acentuado aún más y porque nos parecía que su cuerpo de alguna manera empezaba a flotar entre el cielo y la tierra!

2ํㅡㄹ Reducción de la movilidad: junto con el alejamiento de los afanes del mundo, observamos en el hombre mayor una especie de pobreza de movimientos. Esos movimientos que acompañan naturalmente a una acción determinada, y que no son del todo necesarios, van desapareciendo poco a poco con la edad. Mientras el hombre joven puede al mismo tiempo caminar, conversar, saludar y cambiar el paquete que lleva de una mano a la otra, el anciano sólo puede realizar estos distintos movimientos en forma secuencial. Esta motórica propia del envejecimiento denota una inconfundible semejanza con lo que ocurre en la enfermedad de Parkinson. La rigidez, la hipomimia y la ausencia de movimientos expresivos accesorios, que caracterizan a esta enfermedad, serían una versión extrema de esta reducción motora y por ende espacial, propia del hombre anciano. Pero la coexistencia de una reducción del espacio con un alejamiento del mundo se da, paradójicamente, junto a una apertura hacia el espacio más amplio, que es el espacio trascendente, el espacio sagrado. Es frecuente observar un aumento de la religiosidad en los ancianos, lo que no tiene que ver con el miedo a la muerte. Al 
igual que en el joven, la muerte es vivenciada por el anciano como algo muy lejano, aunque en su caso esté muy próxima desde el punto de vista cronológico. La mayor religiosidad del anciano se debe justamente a las características propias de su espacialidad: el alejamiento de los afanes del mundo, la disminución de la motilidad, que de algún modo prepara para el recogimiento y la apertura de la mirada hacia el espacio de la trascendencia.

3o Coexistencia de lo esencial y lo accesorio: el mundo del anciano está lleno de pequeñeces, de objetos inútiles, de órdenes innecesanios, de colecciones cualesquiera. Esto es muy evidente en la senilidad y en particular en la demencia de tipo Alzheimer, pero también se lo ve en el senescente nomal. Las pequeñas cosas adquieren una importancia desmesurada. Goldstein ${ }^{5}$ ha atribuido este rasgo a una pérdida de la actitud abstracta» Pensamos que no es necesariamente así, porque se trata de un fenómeno selectivo. No a cualquier objeto se aferra el anciano como si fuera lo más importante en el mundo, sino a todo aquello que ha tenido una particular significación en su vida: fotografías de tiempos pasados, objetos que pertenecieron a su madre o al hijo fallecido prematuramente $y$, luego, objetos personales como una lapicera o un reloj, que a su edad ya no son tan fácilmente comprables como lo fueron en la etapa productiva de su vida. Pero hay otra razón que nos hace pensar que este rasgo no es la mera expresión de una pérdida de la actitud abstracta y es el hecho que este aparente quedar preso en el espacio de lo pequeño y accesonio se da la mano con un fenómeno exactamente contrario, a saber, una gran capacidad para captar y comunicar cosas esenciales, condición que desde antiguo los ha hecho acreedores del calificativo de sabios. En efecto, con el envejecimiento se va produciendo un proceso de sesencialización», vale decir, una mayor capacidad tanto para aprehender valores eternos como para transmitirlos. Todo lo superfluo va siendo dejado de lado. Recordemos que Antonio Machado ${ }^{6}$, en uno de sus poemas más conocidos, manifiesta el deseo de que en las postrimenías de la vida la muerte lo encuentre łigero de equipaje». En este mismo sentido, no deja de ser curioso el hecho de que con los años, la mayoría de las personas vaya perdiendo peso y que los ancianos obesos sean una excepción. Es como si la naturaleza misma se hiciera parte de este proceso de aligeramiento»del cuerpo y del espacio, necesario en las cercanías de la muerte.
En resumen, la espacialidad del senescente está marcada por tres parejas de elementos opuestos: cercanía y distancia; reducción del espacio hasta la casi inmovilidad en la enfermedad de Parkinson y apertura hacia el más amplio de los espacios, el espacio sagrado; fijación a lo pequeño $\mathrm{y}$ accesorio de los objetos del entorno y, al mismo tiempo, capacidad de captar cosas esenciales y luego de transmitirlas.

\section{b) La temporalidad de la senescencia}

Con el tiempo ocurre como con el espacio, así, el tiempo cronológico es sólo un derivado, una abstracción del tiempo existencial. Frente a la uniformidad del tiempo cronológico en la vida humana, existen distintos tiempos según la edad o las circunstancias. El tiempo de la alegría es distinto al tiempo del dolor, el de la espera es infinitamente largo, mientras corto se hace siempre el tiempo del encuentro feliz. ¡Con qué velocidad transcurre en las etapas creativas de la vida y cuán lento se hace en la depresión! Pero también las distintas etapas de la vida tienen su propia temporalidad. Nadie podría discutir que el tiempo del niño es muy distinto al tiempo del adulto o al del anciano. Intentaremos delinear las características principales del tiempo de la senectud.

$1^{\circ}$ Enlentecimiento: el niño vive el tiempo como un lento transcurrir y entre el comienzo de las clases y el próximo veraneo, el espacio de tiempo le parece infinito. En el adulto ocurre lo contrario: para él cada año transcurre más rápido y esta velocidad aumenta en directa relación con los proyectos, realizaciones, compromisos y viajes que tenga. En la vejez, en cambio, abandonado ya el trabajo y sin tener que esforzarse para ganar el sustento, el tiempo vuelve a enlentecerse $\mathrm{y}$, por ende, vuelve a alejarse la muerte, esa situación límite que tanto se acercara en la edad adulta, bajo la forma del miedo o la angustia y cuando nos encontrábamos en medio de las responsabilidades de hijos y trabajo. En la vejez nada urge y nadie nos necesita como antes en la oficina o en la casa, pero tampoco nadie nos obliga ni nos manda. Volvemos a tener entonces la sensación infantil de la omnipotencia: cualquier día haremos esto o aquello.

$2^{\circ}$ Contemporaneidad de momentos esenciales y triviales: paralelamente con este enlentecimiento ocurre otro fenómeno característico. Cada momento adquiere una importancia muy grande y casi 
equivalente. Es como si el anciano perdiera la capacidad de distinguir entre los momentos que son esenciales y los que no lo son y así, él da la misma importancia a la pérdida de un objeto cualquiera que a la visita de un hijo o a los síntomas iniciales de una enfermedad. Una versión extrema de este fenómeno es la conocida reacción catastrófica»que presentan los ancianos con signos de deterioro o demencia frente a sobre-exigencias o frustraciones de la vida cotidiana. Sin distinguir el distinto valor o importancia que pueda tener una situación u otra, él reacciona con la misma angustia e irritación. Este fenómeno tiene, empero, su lado positivo y es el nivel de entrega que el anciano puede alcanzar en la relación con sus nietos y parientes, pero también con los animales. Quien haya tenido la suerte de gozar largamente a sus abuelos podrá dar fe de lo antedicho. ¡Cuánto tiempo nos dedicaron en la niñez, como si nosotros fuésemos lo más importante del mundo para ellos! En la literatura hay numerosos ejemplos de descripciones sobre la infinita riqueza que pueden alcanzar determinados momentos junto a personas ancianas.

$3^{\circ}$ La presentización: el futuro se esfuma al no haber obligaciones que cumplir ni proyectos que realizar. Toda la fuerza que el joven y el adulto invierten en un futuro que todavía no existe, se concentra en el anciano en el momento presente, haciéndolo más rico, intenso y prolongado. Por su parte, el pasado, tan olvidado por jóvenes y adultos, se hace palpablemente presente en el anciano a través de esa extraordinaria memoria para los hechos remotos, la que más que una mera capacidad mecánica de revivir imágenes, es otra forma de estar en el mundo y orientarse en él. Esta presentización del pasado nos explica esa capacidad del anciano de entregarse por horas a un presente sin actividades ni logros concretos, a un presente en apariencia vacío. En esos momentos, el anciano vive intensamente recuerdos lejanos, repasa minuto a minuto lo que fuera su niñez, su juventud y primera madurez y aprovecha toda la fuerza vital que el adulto gasta en proyectos para vivir con redoblada intensidad y a veces también profundidad, en ese presente que tiene ahí delante: un nieto, un paisaje, una conversación. Quizás sea este fenómeno de la concentración de toda la fuerza vital en un presente penetrado del pretérito, $\mathrm{y}$ alejando todo sueño sobre futuros inalcanzables, lo que otorga al anciano esa consistencia y redondez que en algunos alcanza el nivel de la sabiduría. El origen de la palabra sabiduría es sapere, que en latín significaba originalmente, saber en el sentido de saborear. Sabio era en el mundo clásico aquel que sabía distinguir sabores de vinos y comidas. El sabio es entonces un catador o un gourmet. Ahora bien, si algo es propio de estas personas es su capacidad de dar con la medida justa, con la medida perfecta, algo que tiene en común con el hombre de buen gusto, que lo emparienta con el hombre de sentido común. El sabio es todo esto en forma superlativa. El anciano llega naturalmente a la sabiduría desde este fenómeno de la presentización, a través del cual podrá saborear bien y descubrir la medida y el buen gusto de ese presente concentrado y enriquecido que es 0 debería ser su vida.

En resumen, la temporalidad del senescente está marcada también por tres parejas de elementos opuestos: enlentecimiento versus la agitación, como respuesta a la sobre-exigencia o la frustración; preocupación excesiva por momentos 0 situaciones triviales versus capacidad de captar y transmitir cosas esenciales; capacidad de vivir intensamente un presente concentrado versus la desorientación que implica un excesivo desprendimiento con respecto al pasado y al futuro.

\section{FenOMENOLOGÍA DE LA DEMENCIA}

En el envejecimiento patológico o senilidad, todos los rasgos descritos pierden su aspecto positivo para mostrar sólo el lado deficitario. Y así, en lo que se refiere a la espacialidad, el «alejamiento»se transforma en indiferencia, la seducción de la movilidad», en inmovilidad y pérdida del acceso al espacio trascendente, mientras la «coexistencia de lo esencial y lo accesorio» se transforma en una mera ocupación con objetos inútiles y órdenes innecesarios. Por su parte, en lo que se refiere a la temporalidad, el <enlentecimiento»se transforma en una paralización del devenir temporal, la «contemporaneidad de elementos esenciales y triviales» desparece bajo el imperio de la trivialidad y la spresentización» o presente enriquecido se diluye hacia una puntualidad vacía sin conexión alguna con el pasado ni con el futuro. 
Si prescindimos de las distintas formas de demencia según su etiología (enfermedad de Alzheimer, demencia vascular, demencia post-traumática, demencia alcohólica, etc.) y nos atenemos sólo a las formas de presentación de los síndromes orgánicocerebrales, tendríamos que distinguir, siguiendo a Scheller ${ }^{7}$, al menos tres grandes síndromes: un síndrome cerebral difuso», descrito fundamentalmente por Bleuler ${ }^{9}$, un síndrome cerebral focal con varios subsíndromes, descrito por Scheller y el síndrome de Korsakoff. Las causas más frecuentes del «síndrome cerebral difuso», son la enfermedad de Alzheimer y la demencia por infartos múltiples 0 vascular y los síntomas fundamentales son el compromiso de la memoria, en particular de la de fijación, la alteración del pensamiento, en el sentido de una disminución de la cantidad de representaciones simultáneamente posibles y la alteración de la afectividad, caracterizada en lo fundamental por la incontinencia afectiva y las reacciones catastróficas. A lo anterior, habría que agregar el cambio de la personalidad, que va a ser diferente en intensidad y forma según la etiología.

El síndrome cerebral focal»muestra, en primer plano, alteraciones de la afectividad, de la impulsividad y de la espontaneidad, con una conservación relativa de la inteligencia y de la memoria. $\mathrm{H}$ Scheller ha distinguido tres subsíndromes focales: el «síndrome de la pérdida de los valores»), descrito originalmente por Meynert, y donde el daño reside en la región basal del lóbulo frontal 0 cerebro orbital de Kleist. Las causas más importantes son la enfermedad de Pick y los meningiomas de la región olfatoria. El segundo subsíndrome de Scheller, es el de la pérdida de la espontaneidad», cuadro en el cual los actos libres y orientados hacia un propósito son reemplazados paulatinamente por actos cada vez más automáticos, que obedecen en forma ciega a estímulos externos. Este síndrome aparece cuando se compromete la parte anterior del lóbulo frontal, ya sea por un tumor, por un traumatismo o por la misma enfermedad de Pick. Este cuadro se combina frecuentemente con el síndrome cerebral difuso en los cuadros demenciales de tipo Alzheimer muy avanzados. El tercer subsíndrome, es el de la pérdida de la capacidad simbólica», con asimbolia y afasia transcortical y que se presenta en lesiones localizadas en el lóbulo temporal. Este subsíndrome aparece en las formas preseniles de la enfer- medad de Alzheimer o como consecuencia de traumatismos encéfalo-craneanos.

La tercera forma de presentación de los cuadros demenciales es el síndrome de Korsakoff, que ha de considerarse como independiente, tanto del síndrome cerebral difuso como del local, por tener un perfil psicopatológico muy característico. Los síntomas clásicos de este síndrome son la amnesia para hechos cercanos en el tiempo con conservación de los recuerdos antiguos y también de la memoria de fijación inmediata; la desorientación témporo-espacial; la fabulación de perplejidad y los falsos reconocimientos. El sustrato anátomo-patológico del síndrome de Korsakoff es la lesión bilateral, aunque no necesariamente simétrica, del llamado circuito de Papez o circuito límbicohipocampo-mámilo-tálamo-cingular. Las causas más frecuentes de este síndrome son el alcoholismo, las intoxicaciones severas con gases como el monóxido de carbono y el anhídrido sulfuroso y los tumores que pueden afectar esa zona.

La pregunta es ¿qué constituye lo esencial de la demencia? ¿Por qué hablamos de demencia tanto en el «síndrome cerebral difuso»de Bleuler ${ }^{8}$, con el típico compromiso de la memoria, la inteligencia y la afectividad y también en los síndromes cerebrales focales» de Scheller ${ }^{7}$, donde, a veces, la memoria y la inteligencia en gran medida se conservan? Lo que todos estos cuadros presentan en común es un cambio global de la personalidad, que hace que los familiares consideren al paciente como esencialmente «distinto». Este carácter global del cambio de la personalidad, no en el sentido de los «rastornos de personalidad», sino del ser-persona, que observamos en las demencias, también ocurre en una enfermedad completamente distinta, como es la esquizofrenia. Ambos cuadros también tienen en común el carecer de conciencia de enfermedad: ni el esquizofrénico acepta estar loco ni discutir el contenido de su delirio, ni el demente se da cuenta de su empobrecimiento intelectual, afectivo 0 valórico. Uno podría pensar, entonces, que en ambas enfermedades hay una falla en la sconciencia de sí mismo» Sin embargo, algo nos dice que el fenómeno es diferente en una y otra, y precisar esta diferencia podría ser una forma de acercarnos a la esencia misma de la demencia. Porque cabe preguntarse: ¿el hecho que un esquizofrénico afirme que le transmiten pensamientos por telepatía, significa necesariamente que él carece de la capaci- 
dad de reflexionar sobre sí mismo, o no es más bien que el mundo se le abre a su percepción conteniendo la posibilidad de la transmisión telepática y que al ser ese el modo de su mundo no cabe que tenga dudas sobre él? Recordemos la famosa frase de Hegel: $\mathbb{E l}$ individuo es lo que es su mundo en cuanto suyo». El esquizofrénico se asume y se proyecta como ser histórico, vale decir, como persona, pero lo hace hacia un mundo que para el resto de la sociedad es extraño, absurdo, imposible, mágico, o lo que sea. De lo que resulta que, la tal falta de conciencia de enfermedad que le atribuimos al paciente psicótico nada tiene que ver con su conciencia de sí como tal, sino más bien con el hecho de una incompatibilidad de mundos que se chocan: el del esquizofrénico y el de los otros, los sanos, ipero mundos, al fin!

Muy distinto se nos muestra el fenómeno de la falta de conciencia de enfermedad en el demente. Cuando el enfermo de Korsakoff mira nuestro delantal blanco y el tubo de luz neón y nos confunde con su amigo, el carnicero de la esquina, resulta evidente que lo que él no está pudiendo hacer, es tomar distancia de su vivenciar irreflexivo y apegado a su entorno para definirse o más bien asumirse respectivamente, por ejemplo, como un sujeto de 75 años con tal o cual historia, que ha venido a consultar a un médico por determinadas molestias, a pedir su ayuda, etc. Es decir, el paciente orgánico equivoca, y con toda naturalidad, la situación y la identidad del médico desde su incapacidad de colocarse sobre la situación vivida, para reflexionar acerca de ella y de sí mismo en cuanto sujeto histórico. Es por eso que su vivenciar es el mero reflejo de las necesidades vegetativas 0 de las emociones elementales, mientras su conducta termina por reproducir automáticamente, en los fenómenos en «eco», los estímulos externos, como ocurre en algunos síndromes cerebrales locales. En el esquizofrénico, en cambio, hay una metamorfosis semántica de un mundo ya trascendido por un yo percipiente y configurante y en esa medida es que podemos afirmar que su auto-reflexividad está conservada. Zutt ${ }^{10}$ piensa que la capacidad de reflexión sobre sí mismo constituye la función más elevada y más propia de la inteligencia humana, mientras que el resto de las habilidades las tenemos casi todas en común con los animales. El demente ha perdido la capacidad de saber que sabe o no sabe, que puede 0 no puede, que ha vivido o no ha vivido y, por eso, no recuerda, no entiende, es desajustado, es torpe, es esclavo del mundo en cuanto estímulo. Esta forma superior de inteligencia corresponde a lo que también se conoce como sactor $G$ de Spearman», factor que satura o trasciende todas las pruebas y que, según Henry Ey, no es sino <el yo (mismo) en cuanto sujeto y agente de la inteligibilidad de su mundo»1.

Es la capacidad de conocerse conociendo el mundo, y viceversa, la que se pierde en los cuadros orgánico-cerebrales que conducen a la demencia. Así concebida esta cualidad superior del yo, se comprende la afirmación de Brinck ${ }^{12}$, cuando dice que la conciencia es una cualidad de los actos, porque el yo es en cuanto actualización y cada acto lo sé mío y al saberlo lo trasciendo y conozco y me conozco, sea que a través de mi acción modifico el entorno o que éste me modifica a mí a través de la percepción. Pero soy siempre yo quien opera y sabe que opera 0 percibe y sabe que percibe. Al fracasar esta función fundamental, por la cual el yo se constituye a sí mismo en las actualizaciones, desaparece necesariamente la memoria, por cuanto lo que se recuerda es justamente esa conciencia del acto; pero también se pierde la capacidad de abarcar la situación como totalidad (pérdida de la actitud categorial) y las emociones pierden su nexo esencial con el acto inteligente para reducirse a expresiones fragmentarias y desproporcionadas.

En los síndromes cerebrales locales también se puede comprender la mayor parte de los síntomas como expresión de la pérdida de la conciencia de sí mismo. Y así, en el síndrome de la pérdida de los valores», también llamado demencia afectiva, el yo se presenta como un mero efector de necesidades vegetativas elementales, desapareciendo toda referencia a esa región del mundo del deber-ser, hacia el cual el yo venía encaminándose desde la oscuridad biológica de la infancia, a través de su permanente trascender la expeniencia vivida. No tener valores significa incapacidad de proyectarse más allá de lo inmediato, esclavitud frente a las pulsiones internas. En el «síndrome de la pérdida de la espontaneidad» ocurre, en cierto modo, lo contrario, pues la existencia se hace esclava de los estímulos extemos, hasta el extremo de la aparición de ecolalia y ecopraxia. El yo ya no puede comandar el vivenciar ni modificar el entomo, porque toda posibilidad de elevación sobre la situación, toda trascendencia se 
ha hecho imposible y así la vida psíquica deviene en una caricaturesca repetición de lo que aparece en el campo de la conciencia, sea como pulsión intema o como estímulo externo.

En suma, todos los déficits constatados en las distintas formas de demencia pueden reducirse a una sola falla fundamental: la incapacidad del yo de volver sobre sí mismo y tomar una posición 0 actitud, elevándose por encima de la vida prereflexiva. Pero ocurre que esta capacidad del yo no es una función más, sino aquello que define al hombre como ser racional e histónico. Si seguimos el pensamiento de Teilhard de Chardin ${ }^{13}$, descubrimos que lo que pierde el demente es, justamente, la capacidad que surge como clímax del proceso de la evolución, allí donde la naturaleza se vuelve, se enrolla sobre sí misma y da lugar a la conciencia a través de una sola de sus especies, el homo sapiens. Pero al mismo tiempo, esta capacidad de reflexión, este volver sobre sí mismo, es lo más propio del concepto que más acertadamente ha definido al ser humano, cual es el de persona. En la dialéctica entre el sujeto y su máscara, implícita en el concepto de persona, propuesto en la Grecia clásica, late esta conciencia de sí, distinta, pero simultáneamente idéntica al sujeto viviente y que hace del enfermo orgánico cerebral, al faltar, un demente. Ser demente significa entonces dejar de ser persona, algo que jamás se podnía decir del esquizofrénico o del más grave de los depresivos. El esquizofrénico puede ser absolutamente autista o amanerado o incomprensible, pero sigue siendo una persona, en la medida que se asume de alguna manera y proyecta un mundo, se opone al otro y se sabe histónico, vale decir, está orientado. Porque la desorientación temporal, rasgo característico de todas las demencias, no es una consecuencia necesaria de la amnesia, como se afirma en algunos textos, ni menos aún de una agnosia particular, sino el resultado de haber perdido la capacidad de ser y por tanto, de hacer historia.

\section{CONSECUENCIAS ÉTICAS}

\section{a) Relativas a la senescencia}

Definida la ancianidad como una nueva etapa de la vida, con desafíos, proyectos $\mathrm{y}$, sobre todo, ventajas sobre otras etapas de la vida, la primera consecuencia ética será abandonar los prejuicios que pesan sobre la vejez, reconociendo la digni- dad de sus diferencias. El prejuicio más generalizado es que el anciano está sometido inevitablemente a un proceso regresivo y destructor, cuyo término es la muerte. Sin embargo, sabemos que no hay un parámetro biológico característico de la vejez, que no existe una correspondencia entre los hallazgos imaginológicos y la inteligencia o el rendimiento de los ancianos y, por último, que hay muchísimos casos de ancianos que no se demencian y que mantienen su espíritu despierto hasta el final. Un ejemplo concreto de prejuicio sin fundamento se refiere a una de las características fenomenológicas que describiéramos en la vejez: el enlentecimiento. En efecto, todo en ellos es más lento y el tiempo mismo, como decíamos, vuelve a fluir con la morosidad de la infancia. Pero ocurre que las personas ancianas compensan largamente esa menor velocidad con la mayor concentración que son capaces de lograr. Ellos cometen muchos menos errores de distracción que los jóvenes. Es, con frecuencia, la sociedad la que le impone al anciano una velocidad que no se condice con la que es propia de su edad. Esto sucede a diario con la velocidad de cierre de las puertas de los trenes del metro, con el tiempo que se les exige para hacer un depósito o una gestión bancaria cualquiera, etc. La sociedad debe, ante todo, conocer lo que es la ancianidad en sus aspectos, tanto positivos como negativos y acomodar en lo posible los tiempos y los espacios urbanos a la temporalidad y a la espacialidad de estos seres privilegiados que son los ancianos.

Otro estereotipo negativo es el que se refiere a su capacidad afectiva. Se les supone incapaces de establecer nuevas relaciones afectivas o amorosas. Lo que ocurre de verdad, es que dentro de este estrechamiento de la existencia propio de la edad disminuye el interés por aumentar el círculo de relaciones; pero, en cambio, su capacidad de profundizar en ellas es mucho mayor.

En el campo cognitivo y aun cuando en el mundo moderno se los tiende a marginar, es sabido que a lo largo de la historia su situación ha sido diferente. En la antigüedad clásica se les tenía el mayor respeto y se les identificaba casi con la sabiduría. Recordemos la Asamblea de los Gerontes, en Grecia, o una institución que llega hasta el día de hoy, como es el Colegio de Cardenales en Roma, donde la mayoría de sus miembros ha sobrepasado los 70 años. 
Los ejemplos de malentendidos respecto a la vejez son múltiples y no es el caso de extenderse más. Pero consideramos fundamental otorgarle al anciano el lugar que le corresponde en la sociedad, reconociendo sus muchos aspectos positivos y tratando de compensar como sociedad sus fragilidades y sus déficits.

\section{b) Relativas a la demencia}

La demencia plantea un problema ético completamente diferente a la senescencia, por cuanto se pierde justamente lo más propio de la condición humana: la auto-reflexión y el manejo de sí mismo y del entorno. La tarea fundamental de la sociedad es, entonces, la protección del demente, porque él no es capaz de cuidar de sí mismo en ningún sentido. Las preguntas claves son ¿en qué momento declararlo interdicto y nombrarle un tutor?, ien qué momento trasladarlo desde la casa familiar a la casa de reposo?, tomando en consideración que los cambios son para ellos muy perturbadores. Ahora bien, esto no significa, en casos de demencia muy extrema, que la familia tenga que mantenerlo en casa hasta el último minuto. Dependerá mucho de las condiciones económicas y del número de familiares que puedan encargarse del cuidado del paciente demente, pero, en nuestra opinión, un buen momento para promover el

\section{REFERENCIAS}

1. PeLICIER Y. Les rôles du troisième âge: Un indicateur socioculturel d'efficacité. Sciences et Devenir de l'Homme, Les Cahiers du MURS 1985; 2: 19-33.

2. DöRR-Zegers O. Approche phénomenologique au problème du vieillissement normal. 1993; 25, 13: 1319-23. Psychologie Medicale

3. Heidegger M. Sein und Zeit (1927). 10. Auflage. Tübingen: Niemayer, 1963.

4. Buytendijk FJJ. Allgemeine Theorie der menschlichen Haltung und Bewegung. Berlin-GöttingenHeidelberg: Springer Verlag, 1956.

5. GoLdTEin K. Zum Problem der Tendenz zum ausgezeichneten Verhalten. Dtsch. Z. Nervenheilk 1929; 109: 1-61.

6. Machado A. «etrato». En: Antología Poética. Estella: Salvat Editores S. A.; 1971. traslado a una institución especializada es cuando ya se instala definitivamente la incontinencia de esfínteres. Esto es válido en lo fundamental para los síndromes cerebrales difusos y, en particular, para los pacientes con Alzheimer, los que, como es sabido, mantienen las formas de conducta y de la educación recibida hasta bastante avanzada la enfermedad. Distinto es el caso en los síndromes frontales o de pérdida de los valores, donde el comportamiento del paciente puede hacerse inmanejable al interior de la familia, dada la posibilidad de conductas perversas y agresivas. Pero, en todo caso, así como en la senescencia el acento tiene que ser puesto en permitirle el mayor desarrollo posible de sus potencialidades, en el caso de los cuadros demenciales será lo más importante el aspecto custodial, que deberá ser, por cierto, lo más elaborado y digno posible, no en último término, porque al representar la demencia el mayor fracaso imaginable del espíritu humano genera necesariamente, en los que todavía no están dementes, miedo, angustia y hasta diría, pavor. Mayor debe ser entonces la preocupación por no caer en actitudes de desprecio y abandono hacia seres que, aun cuando han perdido lo mejor de sí mismos, representan una triste posibilidad humana de la que ninguno de nosotros puede declararse libre.

7. Schejer H. Über den Begriff der Demenz und unterscheidbare klinische Formen von Demenzen. Der Nervenarzt 1965; 36: 1-7.

8. Bleuler E, Bieuler M. Lehrbuch der Psychiatrie. Berlin, Heidelberg, New York: Springer-Verlag; 1975.

9. Hegel GWF. Cit. por Blankenburg, W., en: Der Verlust der natürlichen Selbstverständlichkeit. Stuttgart: Ferdinand Enke Verlag; 1971; 116-17.

10. ZutT J. «Was lehren uns die Demenzzustände über die menschliche Intelligenz». Der Nervenarzt 1964; 35: 1-5.

11. Ey H. Tratado de Psiquiatría. Barcelona: Editorial Toray-Masson, S. A. (1969).

12. BRINck G. Síntomas comunes en el cuadro orgánico cerebral. Santiago: Ediciones de la Vicerrectoría de la Universidad de Chile; 1974.

13. Tellhard de Chardin P. El fenómeno humano. Madrid: Taurus; 1967. 